\title{
Brief Pain Inventory
}

National Cancer Institute

\section{Source}

National Cancer Institute. Brief Pain Inventory. NCI Thesaurus. Code C18203.

A tool for the assessment of pain in both clinical and research settings. The BPI uses simple numeric rating scales from 0 to 10 that are easy to understand and easy to translate into other languages. On the BPI, mild pain is defined as a worst pain score of 1 -4 , moderate pain is defined as a worst pain score of $5-6$, and severe pain is defined as a worst pain score of 7-10. 